\title{
INSETICIDAS UTILIZADOS PARA O CONTROLE DE MOSCA-DAS-FRUTAS EM POMARES DE MACIEIRA: AÇÃO SOBRE Neoseiulus californicus (ACARI: PHYTOSEIIDAE)
}

\author{
INSECTICIDES USED TO CONTROL FRUIT FLIES IN THE APPLE \\ ORCHARDS: ACTION ON Neoseiulus californicus (ACARI: PHYTOSEIIDAE)
}

\author{
Lino Bittencourt MONTEIRO ${ }^{1}$
}

\begin{abstract}
RESUMO
O objetivo do estudo foi avaliar o efeito dos inseticidas malathion, azinphos ethyl, phosmet e fenthion sobre duas populações de Neoseiulus californicus (McGregor). A primeira população foi criada em bandejas (PCB) e não recebeu qualquer inseticida durante, pelo menos, dois anos, sendo que a segunda ficou exposta aos inseticidas normalmente utilizados contra Anastrepha fraterculus (Wied) e denominada de população liberada no pomar (PLP). Vinte e quatro fêmeas de idades conhecidas e de ambas as populações foram colocadas em 12 células de $2 \times 2 \mathrm{~cm}$ e foram alimentadas diariamente com adultos de Tetranychus urticae Koch. Elas foram pulverizadas com um atomizador de laboratório, marca Desaga (Heidelberg), $\mathrm{n}^{\circ} 124000$, que proporciona pulverização fina e homogênea. As avaliações de oviposição e mortalidade foram realizadas após 12, 24, 72 e 96 horas da pulverização. Phosmet e malathion provocaram 50 e $66 \%$ de mortalidade de $N$. californicus das PLP e PCB, respectivamente, na concentração recomendada para macieiras, enquanto que, fenthion e azinphos ethyl foram muito tóxicos para ambas populações, provocando $100 \%$ de mortalidade na concentração de $1.500 \mathrm{ppm}$. Concluiu-se que a PLP sobre pressão de inseticidas tornou-se mais tolerante aos produtos phosmet e malation, que a PCB, em apenas um ciclo vegetativo da macieira.
\end{abstract}

Palavras-chave: Phytoseiidae, ácaro predador, resistência, macieira.

\begin{abstract}
The objective of this work was to evaluate the effect of insecticides malathion, azinphos ethyl, phosmet and fenthion about two populations of Neoseiulus californicus (McGregor). The first population was reared in the tray $(P C B)$ and not recevied any inseticide during two year and the second population was released in the orchard (PLP) and its development was influenced to the insecticides for the control of Anastrepha fraterculus (Wied). Twenty four females of both populations of known ages was reared inside of twelve cells of $2 \times 2 \mathrm{~cm}$ and were fed females of Tetranychus urticae (Koch). The insecticides were sprayed with a Desaga atomizer, $n^{\circ} 124000$ (Heidelberg) that spreaded a fine and homogeneous spraying. Assessment of oviposition and death was done after 12, 24, 72 and 96 hours. Phosmet and malathion killed 50\% and $66 \%$ of $\mathrm{N}$. californicus of PLP and PCB, respectively in the advisable concentration for apple, while was observation with fenthion and azinphos ethyl in the 1.500 ppm concentration were very toxic, and ovoposition did not ocurr on both populations. The conclusion was that the PLP under insecticide pressure was more tolerant to phosmet and malathion than PCB, under apple orchad conditions during five months.
\end{abstract}

Key words: Phytoseiidae, predator mite, resistence, apple.

\footnotetext{
1 Engenheiro Agrônomo, Doutor, Universidade Federal do Paraná, Professor Adjunto, Departamento de Fitotecnia e Fitossanitarismo, Caixa Postal 19061, CEP 80531-990, Curitiba, PR.
} 


\section{INTRODUÇÃO}

Na cultura da macieira ocorrem o ácaro vermelho Panonychus ulmi (Acari: Tetranychidae), a mosca-dasfrutas, Anastrepha fraterculus (Lepidoptera: Tethritidae), a mariposa oriental Grapholita molesta (Lepidoptera: Tortricidae) e a lagarta enroladeira Bonagota cranaodes (Lepidoptera: Tortricidae), que são controlados, quase que exclusivamente, com inseticidas e acaricidas (Bleicher et al.,1982 [1]; Kovaleski, 1998 [5]; Salles, 1995 [10]). Embora as pulverizações sejam freqüentes durante o ciclo vegetativo da cultura, o número de tratamentos tem aumentado nos últimos anos em função do aumento das populações das pragas.

A mosca-das-frutas é uma das pragas que tem apresentado eficiente controle, tendo em vista que os agroquímicos utilizados apresentam tanto ação de profundidade, que evita o desenvolvimento de larvas no interior das maçãs, quanto de contato, que proporciona o controle dos adultos (Salles, 1991 [10]). Os mesmos agroquímicos que são eficientes para as pragas apresentam, entretanto, alta toxicidade para os inimigos naturais (Gutierrez, 1981 [4], Monteiro, 1993 [7]), o que possivelmente, tem colaborado para o aumento das populações de artrópodos pragas.

Entre os inimigos naturais mais importantes em macieira, os ácaros predadores têm sido o principal agente biológico controlador de $P$. ulmi, cujas presenças nos pomares são favorecidas pelo uso de agroquímicos seletivos. Neoseiulus californicus (Acari: Phytoseiidae), espécie cujo centro de origem é o Peru, foi introduzida em um pomar de macieira em Vacaria com a finalidade do controle biológico do ácaro mostrando os efeitos de agroquímicos sobre esta espécie de ácaro predador.
O objetivo do estudo foi avaliar os efeitos de inseticidas mais utilizados para o controle de $A$. fraterculus, sobre duas populações de $N$. californicus.

\section{METODOLOGIA}

A partir de indivíduos adultos de $N$. californicus, provenientes da criação mantida pela EMBRAPACNPMA, foi iniciada uma criação em estufas (Monteiro, 2002) [8], usando como alimento Tetranychus urticae (Acari: Tetranychidae) para liberação nos pomares de macieira da Agropastoril Rincão das Flores, em Vacaria, RS. Entre outubro e dezembro 1996 foram transferidos para o pomar, aproximadamente dois milhões de ninfas e adultos de $N$. californicus em uma área isolada de 50 ha de macieiras da variedade Gala, ou seja, quarenta mil predadores por hectare.

Para a realização dos testes de toxicidade de agroquímicos sobre os ácaros predadores, a população de $N$. californicus foi dividida em dois lotes. O primeiro permaneceu em bandejas de criação sem sofrer qualquer pressão de agroquímicos durante dois anos, sendo denominado de "população criada em bandejas" (PCB). O segundo lote constituiu-se de ácaros predadores coletadas aleatoriamente nos 50 ha de pomar, onde foram liberados os ácaros predadores. Os indivíduos desse segundo lote ficaram expostos aos inseticidas e acaricidas utilizados para o controle de pragas (Quadro 1) entre outubro e fevereiro, sendo denominados de "população liberada no pomar" (PLP).

As populações PCB e PLP foram criadas em laboratório até a obtenção de fêmeas adultas com idade conhecida, as quais foram colocadas em uma arena de plástico preto de 2,0 cm de diâmetro,

Quadro 1 - Inseticidas e acaricidas aplicados no pomar de macieira da variedade Gala (50 ha), Agriflor, Vacaria, RS, 1995/96

\begin{tabular}{|c|c|c|c|c|c|c|c|c|c|c|}
\hline \multirow{2}{*}{ Quadra } & \multicolumn{10}{|c|}{ Data de pulverização do agroquímico } \\
\hline & $30 / 10$ & 09/11 & $01 / 12$ & $12 / 12$ & $17 / 12$ & $22 / 12$ & $30 / 12$ & $07 / 01$ & $20 / 01$ & $10 / 02$ \\
\hline 1 & $C L+A z$ & MA & FE & $\mathrm{PH}$ & PR & FE & $T R+P R$ & $\mathrm{PH}$ & TR & SU \\
\hline 2 & $C L+A z$ & MA & FE & $\mathrm{PH}$ & PR & FE & $T R+P R$ & $\mathrm{PH}$ & MA & SU \\
\hline 3 & $C L+A z$ & MA & FE & $\mathrm{PH}$ & PR & FE & $T R+P R$ & $\mathrm{PH}$ & MA & su \\
\hline 6 & & $\mathrm{MA}+\mathrm{CL}$ & FE & $\mathrm{PH}$ & PR & FE & $T R+P R$ & $\mathrm{PH}$ & TR & SU \\
\hline 7 & & $\mathrm{MA}+\mathrm{CL}$ & FE & $\mathrm{PH}$ & PR & FE & $T R+P R$ & $\mathrm{PH}$ & TR & SU \\
\hline 8 & & $\mathrm{MA}+\mathrm{CL}$ & FE & $\mathrm{PH}$ & PR & $\mathrm{FE}$ & $T R+P R$ & $\mathrm{PH}$ & TR & SU \\
\hline 9 & $C L+A z$ & $\mathrm{MA}+\mathrm{CL}$ & FE & $\mathrm{PH}$ & PR & FE & $T R+P R$ & $\mathrm{PH}$ & TR & SU \\
\hline 11 & $C L+A z$ & $\mathrm{MA}+\mathrm{CL}$ & FE & $\mathrm{PH}$ & PR & FE & $T R+P R$ & $\mathrm{PH}$ & TR & SU \\
\hline 12 & $C L+A z$ & MA & FE & $\mathrm{PH}$ & PR & $\mathrm{FE}$ & $T R+P R$ & $\mathrm{PH}$ & TR & \\
\hline 13 & $C L+A z$ & MA & FE & $\mathrm{PH}$ & PR & FE & $T R+P R$ & $\mathrm{PH}$ & $T R+P R$ & \\
\hline 15 & & $\mathrm{MA}+\mathrm{CL}$ & FE & $\mathrm{PH}$ & PR & FE & PR & $\mathrm{PH}$ & & SU \\
\hline 16 & & $\mathrm{MA}+\mathrm{CL}$ & FE & $\mathrm{PH}$ & PR & FE & $T R+P R$ & $\mathrm{PH}$ & TR & SU \\
\hline
\end{tabular}

Acaricidas: $\mathrm{CL}=$ clofenthezine $; \mathrm{PR}=$ propargite

depositada sobre algodão úmido, que por sua vez estava em uma caixa de polietileno $(13 \mathrm{~cm} \times 7 \mathrm{~cm})$, 
semelhante a utilizada em estudos de biologia (Monteiro, 1991 [6]). Cada caixa constituiu-se em um tratamento formado de 12 arenas, contendo duas fêmeas cada. As arenas de plástico preto foram pulverizadas e posteriormente receberam as fêmeas de $N$. californicus para avaliar os efeitos da toxicidade dos agroquímicos. O experimento foi conduzido em temperatura de $22 \pm 1^{\circ} \mathrm{C}$, UR de $75 \pm 10 \%$ e fotoperíodo de 12 horas.

Quatro inseticidas utilizados para o controle de $A$. fraterculus foram testados sobre os ácaros predadores: malathion - 2.000, 1.000 e 200 ppm; azinphos ethyl - 1.500, 750 e 150 ppm; phosmet $2.000,1.000$ e 200 ppm; fenthion - 1.000, 500 e 100 ppm, cujas concentrações representam, respectivamente, a concentração homologada para uso em macieira (CHPM), $50 \%$ e um décimo da CHPM. As pulverizações foram realizadas com um atomizador para laboratório, marca DESAGA, $n^{\circ} 124000$, Heldelberg, formando uma névoa fina e homogênea. A mortalidade e a oviposição das fêmeas foram observadas 12, 24, 72 e 96 horas após pulverização.

A fim de que se pudesse ter certeza de que os ácaros predadores coletados no pomar (PLP) fossem da mesma espécie que havia sido liberada, realizaram-se três levantamentos para identificação de ácaros, em ervas daninhas e em folhas de macieira. O levantamento foi realizado na área de liberação e nas parcelas periféricas, as quais não haviam recebido $N$. californicus. A primeira coleta foi feita antes da introdução $N$. californicus e as duas outras ao longo do ciclo vegetativo da macieira (janeiro e fevereiro). Nesses levantamentos não foram encontrados ácaros predadores nas parcelas sem liberação, enquanto que nas áreas de macieiras onde haviam sido liberados foram identificadas somente as espécies $N$. californicus, $P$. ulmi e $T$. urticae.

\section{RESULTADOS E DISCUSSÃO}

A mortalidade de fêmeas das populações PCB e PLP foi de 100\% quando submetidas a concentração recomendada para macieira de azinphos ethyl e fenthion. Entretanto, as concentrações duas e dez vezes menores do tratamento azinphos ethyl permitiram que as fêmeas de PLP colocassem 33 e 59 ovos em 96 horas, respectivamente (Tabela 1). Esses dois inseticidas eram freqüentemente utilizados em pulverização total em anos que antecederam o estudo, sendo reconhecidos como inseticidas tóxicos para fitoseídeos (Service Protection Végétaux, 1987) [12]. Monteiro (1993) [7] observou que fenthion pulverizado em macieira causou redução de $N$. californicus permitindo que $P$. ulmi ultrapassasse o nível de dano econômico.

O tratamento com phosmet, na concentração de 2000 ppm, ocasionou redução da PLP de aproximadamente $50 \%$, após 96 horas, enquanto que os predadores da PCB foram significamente reduzidos em 79,1\% (Tabela 1). A oviposição foi 3,1 vezes maior nas fêmeas da PLP que em PCB (Tabela 2), cuja média de posturas foi de 1,1 ovos/dia. Esse resultado indicou que a PCB era ligeiramente tolerante ao phosmet e que, após exposição aos tratamentos fitossanitários no pomar, houve pressão de seleção com aparecimento de gerações mais resistentes (PCP). A seleção da resistência em populações de ácaros predadores devido a pulverizações com inseticidas organofosforados foi observada por vários pesquisadores (Bostanian et al., 1985 [2]; Caccia et al., 1985 [3]; Prokopy e Christie, 1992 [9], Vidal e Kreiter, 1995 [13]). De acordo com a classificação do Service de la Protection des Végétaux (1987) [12] e levando em consideração a mortalidade de predadores submetidos à concentração recomendada para macieira, phosmet foi moderadamente tóxico ao predador $N$. californicus (Tabelas 1 e 2).

Em relação ao inseticida malathion a população PLP foi mais tolerante do que PCB. Mesmo que malathion tenha provocado $66 \%$ de mortalidade na concentração de 2.000 ppm (Tabela 2) observa-se que, para fins práticos, o efeito negativo sobre as populações de $N$. californicus é limitado pois este agroquímico é usualmente utilizado nas bordaduras dos pomares na forma de isca-tóxica para o controle de moscadas-frutas.

\section{CONCLUSÕES}

As populações PLP e PCP de $N$. californicus a partir de 24 horas após o tratamento tiveram 100\% de mortalidade quando submetidas a fenthion e a azinphos ethyl na concentração recomendada para o controle de $A$. fraterculus. A população liberada no pomar (PLP) de $N$. californicus, desenvolvida sob pressão de seleção por inseticidas, tornou-se mais tolerante aos inseticidas phosmet e malathion em apenas cinco meses.

\section{AGRADECIMENTOS}

Ao Sr. Leandro Kolodny, diretor da Agropastoril Rincão das Flores (AGRIFLOR, Vacaria, RS) pelo financiamento do trabalho e ao técnico Onilton J. Capelini pela ajuda.

\section{REFERÊNCIAS}

[1] BLEICHER, J.; GASSEN, D. N.; RIBEIRO, L. G.; TANAKA, H.; ORTH, A. I. A mosca-das-frutas em macieira e pessegueiro. Florianópolis: Empresa Catarinense de Pesquisa Agropecuária, 1982. $28 \mathrm{p}$. (Boletim Técnico 19). 
TABELA 1 - Percentual de mortalidade de fêmeas de duas populações de Neoseiulus californicus 12, 24, 72 e 96 horas após serem pulverizadas com phosmet, malathion, azinphos ethyl e fenthion, em três concentrações, Vacaria, RS, 1995/96

\begin{tabular}{|c|c|c|c|c|c|c|}
\hline \multirow{2}{*}{ Tratamento } & \multirow{2}{*}{ População } & \multirow{2}{*}{$\begin{array}{c}\text { Concentração } \\
\text { (ppm) }\end{array}$} & \multicolumn{4}{|c|}{ Mortalidade (\%) } \\
\hline & & & 12 horas & 24 horas & 72 horas & 96 horas \\
\hline \multirow{6}{*}{ Phosmet } & PLP $^{1}$ & 2.000 & 0,0 & 0,8 & 41.6 & 50,0 \\
\hline & & 1.000 & 33,3 & 33,3 & 33.3 & 37,5 \\
\hline & & 200 & 41,6 & 41,6 & 41.6 & 41,6 \\
\hline & PCB $^{2}$ & 2.000 & 62,5 & 75,0 & 79,1 & 79,1 \\
\hline & & 1.000 & 33,3 & 50,0 & 58,3 & 66,6 \\
\hline & & 200 & 20,8 & 50,0 & 70,8 & 75,0 \\
\hline \multirow{6}{*}{ Malathion } & PLP & 2.000 & 25,0 & 54,1 & 54,1 & 66,6 \\
\hline & & 1.000 & 20,8 & 58,3 & 62,5 & 70,8 \\
\hline & & 200 & 0,0 & 25,0 & 29,2 & 33,3 \\
\hline & PCB & 2.000 & 87,5 & 100,0 & 100,0 & 100,0 \\
\hline & & 1.000 & 79,1 & 100,0 & 100,0 & 100,0 \\
\hline & & 200 & 95,8 & 100,0 & 100,0 & 100,0 \\
\hline \multirow{6}{*}{ Azinphos ethyl } & PLP & 1.500 & 95,8 & 100,0 & 100,0 & $\overline{100,0}$ \\
\hline & & 750 & 29,1 & 58,3 & 66,6 & 79,1 \\
\hline & & 150 & 20,8 & 37,5 & 37,5 & 54,1 \\
\hline & PCB & 1.500 & 100,0 & 100,0 & 100,0 & 100,0 \\
\hline & & 750 & 100,0 & 100,0 & 100,0 & 100,0 \\
\hline & & 150 & 100,0 & 100,0 & 100,0 & 100,0 \\
\hline \multirow{6}{*}{ Fenthion } & PLP & 1.000 & 100,0 & 100,0 & 100,0 & 100,0 \\
\hline & & 500 & 79,1 & 83,3 & & \\
\hline & & 100 & 66,6 & 79,2 & & \\
\hline & РСВ & 1.000 & 100,0 & 100,0 & 100,0 & 100,0 \\
\hline & & 500 & 100,0 & 100,0 & 100,0 & 100,0 \\
\hline & & 100 & 100,0 & 100,0 & 100,0 & 100,0 \\
\hline
\end{tabular}

TABELA 2 - Número médio de ovos postos por fêmeas sobreviventes de duas populações de Neoseiulus californicus pulverizadas com phosmet, malathion, azinphos ethyl e fenthion, em três concentrações, Vacaria, RS, 1995/96

\begin{tabular}{|c|c|c|c|c|c|c|c|}
\hline \multirow{2}{*}{ Tratamento } & \multirow{2}{*}{ População } & \multirow{2}{*}{$\begin{array}{c}\text { Concentração } \\
(p p m)\end{array}$} & \multirow{2}{*}{ Fêmeas ${ }^{1}$} & \multicolumn{4}{|c|}{ Mortalidade (\%) } \\
\hline & & & & 12 horas & 24 horas & 72 horas & 96 horas \\
\hline \multirow{6}{*}{ Phosmet } & PLP $^{1}$ & 2.000 & 18 & 1 & 22 & 70 & 79 \\
\hline & & 1.000 & 16 & 3 & 23 & 76 & 91 \\
\hline & & 200 & 14 & 3 & 26 & 79 & 89 \\
\hline & $\mathrm{PCB}^{2}$ & 2.000 & 6 & 6 & 14 & 23 & 25 \\
\hline & & 1.000 & 9 & 7 & 22 & 60 & 68 \\
\hline & & 200 & 11 & 12 & 21 & 52 & 61 \\
\hline \multirow{6}{*}{ Malathion } & PLP & 2.000 & 12 & 0 & 17 & 53 & 57 \\
\hline & & 1.000 & 11 & 0 & 27 & 70 & 72 \\
\hline & & 200 & 19 & 0 & 31 & 0 & 97 \\
\hline & PCB & 2.000 & 1 & 0 & 0 & 0 & 0 \\
\hline & & 1.000 & 1 & 0 & 0 & 0 & 0 \\
\hline & & 200 & 0 & 0 & 0 & 0 & 0 \\
\hline \multirow{6}{*}{ Azinphos ethyl } & PLP & 1.500 & 0 & 0 & 0 & 0 & 0 \\
\hline & & 750 & 10 & 0 & 5 & 22 & 33 \\
\hline & & 150 & 15 & 0 & 16 & 47 & 59 \\
\hline & PCB & 1.500 & 0 & 0 & 0 & 0 & 0 \\
\hline & & 750 & 0 & 0 & 0 & 0 & 0 \\
\hline & & 150 & 0 & 0 & 0 & 0 & 0 \\
\hline \multirow{6}{*}{ Fenthion } & PLP & 1.000 & 1 & 0 & 0 & 1 & 1 \\
\hline & & 500 & 2 & 0 & 0 & 1 & 1 \\
\hline & & 100 & 4 & 2 & 4 & 6 & 6 \\
\hline & РCB & 1.000 & 0 & 0 & 0 & 0 & 0 \\
\hline & & 500 & 0 & 0 & 0 & 0 & 0 \\
\hline & & 100 & 0 & 0 & 0 & 0 & 0 \\
\hline
\end{tabular}

${ }^{1}$ Número médio de fêmeas sobreviventes em 96 horas

${ }^{2}$ PLP = População liberada no pomar $\quad{ }^{3} \mathrm{PCB}$ - População criada em bandeja

[2] BOSTANIAN, N. J.; BELANGER, A.; RIVARD, I. ethyl on apple orchard ant their toxicity to Amblyseius Residues of four sinthetic pyrethroids and azinphos- fallacis (Acari: Phytoseiidae). Canadian Entomologist, 
Ontário, n.117, p. 143-152, 1985.

[3] CACCIA, R.; BAILLOD, M.; GUIGNARD, E.; KREITER, S. Introduction d'une souche de Amblyseius andersoni Chant (Acari: Phytoseiidae) résistant à l'azinphos, dans la lutte contre les acariens phytophages en viticultura. Revue Suisse Viticulture Arboriculture Horticulture Lausane, n.17, p. 285-290, 1985.

[4] GUTIERREZ, J. ETIENNE,J. Some data on the tetranychid mites attacking cultived plants in Senegal. Agronomie Tropicale, Paris, v.36, p. 391-394, 1981.

[5] KOVALESKI, A.; BOTTON, M.; EIRAS, A. E.; VILELA E. Lagarta-enroladeira da macieira Bonagota cranaodes (Meyrick, 1937) (Lepidoptera: Tortricidae): bioecologia, monitoramento e controle. Bento Gonçalves: EMBRAPA Uva e Vinho, 1998. 16p. (Circular Técnica 24)

[6] MONTEIRO, L. B. Etude de la relation entre l'alimentation hydrique, la physiologie du pommier et le développement de l'araignée rouge Panonychus ulmi (Koch, 1936) (Acari: Tetranychidae). Montpellier, 1991. $141 \mathrm{f}$. Tese (Doutorado), Ecole Nationale Superieure Agronomique de Montpellier.

[7] MONTEIRO, L. B. Controle biologique de Panonychus ulmi (Koch) (Acari: Tetranychidae) en fonction du programme de traitement contre la mouche des fruits dans la région de Vacaria (Brésil). In: CONFÉRANCE INTERNATIONALE DES RAVAGEURS EN AGRICULTURE 3., 1993, Montpellier. Anais. Paris: ANPP, 1993. p. 611-619.

[8] MONTEIRO, L. B. Criação de ácaros fitófagos e predadores: um caso de produção de Neoseiulus californicus em produtores de maçã. In: PARRA, J. R. P.; BOTELHO, P. S. M.; CORREA-FERREIRA, B. S.; BENTO, J. M. S. (Ed.) Controle biológico no Brasil: parasitóides e predadores. São Paulo: Manole, 2002. p. 351-365.

[9] PROKOPY, R. J.; CHRISTIE, M. Studies on releases of mass-reared organophosphate resist Amblyseius fallacis (Garm.) predatory mites in Massachusets commercial apple orchards. Journal Applied Entomology, Hamburg e Berlin, n.114, p.131-137, 1992.

[10]SALLES, L. A. B. Grafolita (Grapholita molesta) bioecologia e controle. Pelotas: EMBRAPA/CNPFT, 1991. $13 \mathrm{p}$.

[11]SALLES, L. A. B. Bioecologia e controle da moscadas-frutas Sul Americana. Pelotas: EMBRAPA / CNPFT, 1995. $58 \mathrm{p}$.

[12]SERVICE DE LA PROTECTION DES VÉGÉTAUX. Les actions secondaires des produits phytosanitaires. 5.ed. Paris: SPV e ANPP, 1987, 60 p. (Circular 4)

[13]VIDAL, C.; KREITER, S. Resistance to a range of insecticides in the predaceous mite Typhlodromus pyri (Acari: Phytoseiidae): inheritance and physiological machanisms. Journal of Economic Entomology, Maryland, v.88, n.5, p. 1097-1105, 1995.

Recebido para publicação em 06 MAR 2001 [SA 036/2001] Aceito para publicação em 21 AGO 2001 